\title{
Current and Potential Therapies for Ocular Neovascular Diseases
}

\section{Aling Dong*}

Departments of Ophthalmology, The Johns Hopkins University School of Medicine, Maumenee 832, 600 N. Wolfe Street, Baltimore, MD 21287, USA

Diseases complicated by neovascularization and/or excessive vascular leakage in the eye are responsible for majority of vision loss and blindness in developed countries. Diabetic retinopathy (DR) is the most common cause of moderate and severe vision loss among the working age population [1]. Age-related macular degeneration (AMD) is the leading cause of severe vision loss and legal blindness in individuals over the age of 55 [2]. The neovascular AMD leads to significant vision loss in a very short period of time. While there is currently no cure for either of these ocular neovascular diseases, considerable progress has been made in the development of new therapies for these disease process.

To date, there are four types of therapies available for retinal and choroidal neovascularization (NV): angiogenesis inhibitors; Photodynamic Therapy (PDT); laser treatment and Implantable Miniature Telescope (IMT).

The angiogenesis inhibitors officially approved by the Food and Drug Administration (FDA) to treat retinal and choroidal NV, are various Vascular Endothelial Growth Factor (VEGF) antagonisms, including ranibizumab (Lucentis), pepaptanib sodium (Macugen) and aflibercept (Eylea). In addition, bevacizumab (Avastin) has also been used "off-label”. By blocking proangiogenic protein VEGF, these drugs all decrease the abnormal new blood vessels formation and thus reduce the vascular leakage and swelling of the retina. Mounting evidence from randomized clinical trials has confirmed the significant vision improvement efficacy and patient tolerability of ranibizumab [3]. Till now, it has been widely considered the "gold standard" among all antiangiogenic drugs in the treatment of ocular NV. Both developed by Genentech, ranibizumab is a monoclonal antibody fragment (Fab) derived from the same parent mouse antibody as bevacizumab. Approved by FDA in 2006 [4], ranibizumab, a smaller molecule with matured affinity, is designed to be administrated through monthly injections into the eye. As the first commercially available angiogenesis inhibitor, bevacizumab was originally licensed to treat metastatic colon cancer in 2004, and then some other cancers. However it has been widely used off-label for ocular NV due to economic reasons. The landmark Comparison of Age-related Macular Degeneration Treatments Trial (CATT) study has reported that bevacizumab and ranibizumab had equivalent effects on visual acuity after 1 year and through 2 years, when administered according to the same schedule [5]. While it is less costly than ranibizumab, bevacizumab was associated with more Systemic Serious Adverse Events (SAEs) [6].

Another angiogenesis inhibitor, pegaptanib (Macugen) is a pegylated anti-VEGF aptamer, a single strand of nucleic acid that binds specifically to VEGF165. Developed by Eyetech (now OSI Pharmaceuticals) and Pfizer (marketing outside of USA), Macugen gained FDA approval in December 2004. Pegaptanib is administered through injections into the eye, with treatments required every six weeks [7]. The newly approved VEGF inhibitor, Eyelea (aflibercept), is a fusion protein designed to inhibit the action of VEGF in retinal and choroidal NV. Developed by Regeneron and Bayer Healthcare, Eyelea gained FDA approval in November 2011. Also known as VEGF TrapEye, Eylea is recommended to be administrated through injections into the eye every eight weeks (after three initial monthly injections), rather than every four weeks like Lucentis [8].

Visudyne Photodynamic Therapy (PDT) was the first drug therapy approved to treat neovascular AMD. It is a two step procedure involving the intravenous administration of the drug, and subsequent activation by a non-thermal laser beam. This "cool" laser treatment produces a chemical reaction that selectively destroys abnormal blood vessels. However it is only for those patients who have new blood vessel growth under the retina in a well defined, distinctive pattern known as "predominantly classic." Visudyne was developed by Novartis. Laser photocoagulation is another classic treatment for choroidal NV. The procedure uses laser light to destroy or seal off new blood vessels to prevent leakage. A major drawback of PDT and laser photocoagulation is that recurrent NV post-treatment is extremely common, since that these therapies don't eliminate the angiogenic stumili. Laser photocoagulation produces many small retinal scars, which cause blind spots in the patient's visual field. Multiple treatments are always needed.

FDA approved the Implantable Miniature Telescope (IMT) in 2010, developed by VisionCare Ophthalmic Technologies. The intraocular implant magnifies images onto the retina to improve central vision damaged by advanced AMD or Stargardt's maculat dystrophy. This device is only for patients at least 75 years old with severe to profound vision impairment.

Among these treatments, VEGF antagonisms have provided major benefits to patients. Recent progress unveiling the molecular pathogenesis of the diseases has lead to potential new drug therapy. Other key angiogenesis mediators, such as Platelet Derived Growth Factor B (PDGF-B) and NF-kB-responsive mediator Vascular Cell Adhesion Molecule-1 (VCAM-1) [9], can provide potential therapy to combat worsening of NV diseases.

Nonetheless, monthly injections of VEGF inhibitors into the eyes can cause harmful side effects, and some patients didn't have an optimal response. A promising potential new treatment is gene-transfer of antiangiogenic factors through viral vectors, which has the potential to provide long-term suppression of NV and excessive vascular leakage [10].

\section{References}

1. Klein R, Klein BE, Moss SE, Davis MD, DeMets DL (1984) The Wisconsin Epidemiologic study of diabetic retinopathy. II. Prevalence and risk of diabetic retinopathy when age at diagnosis is less than 30 years. Arch Opthalmol 102: 520-526

2. Klein R, Klein BE, Linton KL (1992) Prevalence of age-related maculopathy The beaver dam eye study. Ophthalmology 99: 933-943

3. Frampton JE (2013) Ranibizumab: a review of its use in the treatment of neovascular age-related macular degeneration. Drug Aging 30: 331-358.

*Corresponding author: Aling Dong, Departments of Ophthalmology, The Johns Hopkins University School of Medicine, Maumenee 832, $600 \mathrm{~N}$. Wolfe Street, Baltimore, MD 21287, USA, Tel: 410-502-0808; E-mail: adong1@jhmi.edu

Received June 21, 2013; Accepted June 23, 2013; Published June 25, 2013

Citation: Dong A (2013) Current and Potential Therapies for Ocular Neovascular Diseases. Clin Exp Pharmacol 3: e122. doi:10.4172/2161-1459.1000e122

Copyright: (c) 2013 Dong A. This is an open-access article distributed under the terms of the Creative Commons Attribution License, which permits unrestricted use, distribution, and reproduction in any medium, provided the original author and source are credited. 
Citation: Dong A (2013) Current and Potential Therapies for Ocular Neovascular Diseases. Clin Exp Pharmacol 3: e122. doi:10.4172/2161$1459.1000 \mathrm{e} 122$

4. Genentech (2012) Lucentis: briefing book. U.S.

5. CATT research group (2012) Ranibizumab and bevacizumab for treatment of neovascular age-related macular degeneration: two-year results 119: 13881398.

6. Do DV (2013) Implications of the comparisons of age-related macular degeneration treatments trials on clinical practice: what have we learned? Ophthalmology 120: S8-S10.

7. http://www.fda.gov/newsevents/newsroom/pressannouncements/2004/ ucm108385.htm
8. http://www.accessdata.fda.gov/drugsatfda_docs/label/2011/125387/bl.pdf

9. Dong A, Shen J, Zeng M, Campochiaro P (2011) Vascular cell adhesion molecule-1 plays central role in the proangiogenic effectz of oxidative stress. PNAS 108: 14614-14619.

10. Campochiaro P (2012) Gene transfer for ocular neovascularization and macular edema 19: 121-126. 